\title{
Discrepancy between the Renal Pelvic Fluctuations and the Pacemaker Discharges at the Pelvi-calyceal Border
}

\author{
Seigi Tsuchida and Takashi Morita \\ Department of Urology, Akita University School of Medicine, \\ Akita 010
}

Tsuchida, S. and Morita, T. Discrepancy between the Renal Pelvic Fluctuations and the Pacemaker Discharges at the Pelvi-calyceal Border. Tohoku J. exp. Med., 1983, 139 (3), 329-330 — Experiments were performed in vitro to explore the influence of isoproterenol on the canine pelvi-ureteral system. The rhythmic changes of intrapelvic pressure became no longer manifest and simultaneously recorded electromyograms revealed disappearance of peristalsis at sites distal to the upper region of renal pelvis following application of isoproterenol, while the pacemaker at the pelvi-calyceal border continued to discharge with an enhanced pace. This result has demonstrated that the renal pelvic pressure recorded during treatment with isoproterenol did not reflect the pacemaker activity of the renal pelvis. renal pelvic pacemaker; renal pelvic pressure; renal pelvic electromyogram; isoproterenol administration

Constantinou (1974) observed rhythmic changes of intrapelvic pressure unrelated to the urine output in the in vivo studies on dogs, which, he inferred, might reflect renal pelvic pacemaker control of ureteral peristalsis. We (1981) have demonstrated that the intrapelvic pressure changes invariably corresponded, in a ratio of $1: 1$, with action potentials in the pelvic-calyceal border ( $\mathrm{PC}$ border) in canine in vitro experiments; the observation evidently shows that intrapelvic pressure changes reflect the pacemaker activity. Meanwhile, it is widely recognized that the renal pelvic fluctuation vanished and ureteral peristalsis also diminished or vanished following application of isoproterenol, a $\beta$-adrenergic agent. In view of this, the present study was undertaken to examine detailed responses to isoproterenol of the pacemaker region using an in vitro tracing method we have devised.

The adult mongrel dog was laparotomized under intravenous anesthesia with $15 \mathrm{mg} / \mathrm{kg}$ of thiamylal sodium. The ureter was transected at about $10 \mathrm{~cm}$ distal to the pelvi-ureteric junctin (PUJ), and the kidney was removed along with the proximal ureter. The specimen was then immediately placed in a bath of Krebs-Ringer solution saturated with $95 \%$ oxygen and $5 \%$ carbon dioxide $\left(\mathrm{pH} 7.80,18^{\circ} \mathrm{C}\right)$, in which the renal pelvis was widely exposed up to the extent of calyces by removal of the overlying parenchymal and adipose tissues with the aid of a binocular stereomicroscope. A 4 Fr. two lumen polyethylene catheter was then inserted into the center of renal pelvis through the renal parenchyma. One end of the catheter was connected to an automatic injector for continuous intrapelvic infusion of KrebsRinger solution, and the other end was connected to a Statham P-50 pressure transducer to measure the intrapelvic pressure. Electromyograms were recorded from the PC border, centripelvic region and PUJ by means of aspiration glass microelectrodes, $300 \mu \mathrm{m}$ in outer diameter, which were attached to these specific regions using a micromanipulator. The fluid in the organ bath was replaced with Krebs-Ringer solution containing isoproterenol at concentrations of $10^{-6}$ to $10^{-5} \mathrm{~g} / \mathrm{ml}$ for application of the drug.

Received for publication, July 20, 1982. 
Simultaneous pelvic pressure tracing and electromyograms from the PC border, centripelvic region and PUJ were obtained in six preparations subjected to application of isoproterenol. Fig. 1 shows the tracing before and at $90 \mathrm{sec}$ after isoproterenol application in No. 1 pyeloureter, in which intrapelvic infusion was at a rate of $0.80 \mathrm{ml} / \mathrm{min}$ and the dose of isoproterenol $5 \times 10^{-6} \mathrm{~g} / \mathrm{ml}$. As can be seen, intrapelvic pressure waves $(2.10 \pm 0.06$ sec, $n=18)$ coincided in a ratio of $1: 1$ with the serial discharges in the PC border $(2.12 \pm 0.06$ sec, $n=18$ ) before application of drug regardless of whether or not discharges in the pelvis were blocked, hence obviously reflecting the discharge from the PC border. After application of isoproterenol, in contrast, the baseline of intrapelvic pressure waves declined even in the absence of reduction in intrapelvic infusion rate, and the renal pelvic pressure tracing showed faint waves only on propagation of the PC border action potentials to the region of PUJ. No single discharge from the $\mathrm{PC}$ border was reflected in the intrapelvic pressure waves even in such an instance where the simultaneous tracings revealed clearly action potentials in the PC border alone.

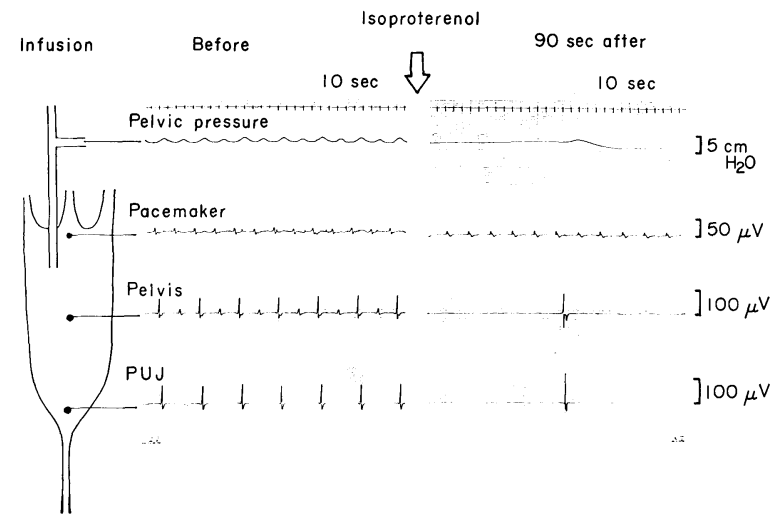

Fig. 1. Simultaneous tracings of intrapelvic pressure and pacemaker and PUJ electromyograms before and after isoproterenol application (in vitro: intrapelvic infusion rate, $0.80 \mathrm{ml} / \mathrm{min})$.

\section{References}

1) Constantinou, C.E. (1974) Renal pelvic pacemaker control of ureteral peristaltic rate. Amer. J. Physiol., 226, 1413-1419.

2) Tsuchida, S. Morita, T., Harada, T. \& Kimura, Y. (1981) Initiation and propagation of canine renal pelvic peristalsis. Urol. int., 36, 307-314. 